Firudin T. Aghayev, Gulara A. Mammadova

Institute of Information Technology of ANAS, Baku, Azerbaijan

DOI: 10.25045/jpit.v07.i1.07 depart10@rambler.ru

\title{
APPLICATION OF THE ANALYTIC HIERARCHY PROCESS IN THE DEVELOPMENT OF OPTIMAL EDUCATIONAL CURRICULUMS FOR THE UNIVERSITIES SPECIALIZED IN ICT
}

The curriculum of the university is a complex system consisting of a set of logical and calendar distribution in disciplines time. The article suggests an approach to the development of educational curriculums by a group of experts. Each of them offers own curriculum version corresponding to a set and procedure of the disciplines. The problem of selecting the most optimal educational curriculum is solved by the analytic hierarchy process.

Keywords: educational curriculum, optimal educational curriculum, criteria for the curriculum evaluation, analytic hierarchy process, calculation of the vector of priorities.

\section{Introduction}

Currently, in accordance with the reforms in the education sector, old curriculums are modernized, renewed and adapted to modern requirements.

Development of educational curriculums for high schools is a complex and challenging task. To implement this task, a set of requirements should be taken into account. At the present, curriculums have to be updated annually, as they remained unchanged before the market economy established in Azerbaijan. The reformation of the education needs a new type of IT professionals, who improve their theoretical and practical knowledge and skills and present creative approach to their work.

Professional training, first, depends on the quality of the educational curriculum of the university, and the structure of the content is defined by a set of subjects and elements of the educational material. Construction of the curriculum structure is based on the general principles of selection of the education content, as well as on the principles of profile of the higher education [1].

All disciplines of the curriculum are interrelated. If we review the curriculum as a set of modules, it can be represented as a graph of relations of educational material. For a strong comprehension of the material, it is necessary to comply with the requirement of consistency of its presentation, that is, the information previously learnt is used during the study of disciplines.

When designing the content, the followings should be considered:

- Calendar time of teaching any section of the discipline must not exceed the prescribed period of study at the university.

- The number of the disciplines studied during the course of education and each semester must not exceed the pre-assigned values.

- The number of teaching hours per week must not exceed the specified standards.

- The intensity of the study of each module must be within the limits specified for the respective academic discipline.

When drawing up the curriculum of university material, the most important profession should be selected and distributed by the semester in an optimal way for learning by following all above requirements.

\section{Selecting criteria for the comparative analysis of educational disciplines}

Selection of the optimal curriculum is possible only based on a set of criteria, according to which they can be compared properly. Taking into account the complexity of the curriculum's structure and a large number of the comparison options with the evaluation criteria, the expert group performs it best. However, the evaluation of curricula on a computer is only possible when all the evaluation criteria will be expressed in numerical form. This will definitely compare them.

In turn, the evaluation criteria must meet certain requirements. The most important ones are 
the followings:

- these criteria must be fully and comprehensively assess the curriculum;

- qualitative indicators to curriculum assessment must comply with modern requirements (innovativeness, relevance and others.);

- all indicators must have a numerical representation for subsequent correct and unambiguous comparisons between them;

- all criteria, if possible, must have analogous "weight" or priorities in order to avoid onesided presentation of the evaluation.

When benchmarking and comparison of academic disciplines, substantiated selection of criteria and priorities in the system of evaluation is very important. Standards and Guidelines for Quality Assurance in the European Higher Education Area [2] are divided into two main groups:

1) Mandatory (formal) criteria take into account the implementation of minimum requirements to the curriculum (e.g., the total number of hours for teaching disciplines).

Implementation of the formal criteria is mandatory, and the curriculum is not considered further without their fulfillment.

2) Qualitative criteria take into account directly the qualitative assessment of the proposed academic discipline that enables their comparison and relative assessment. Among the qualitative criteria the followings are identified:

- compliance with the studied subjects, proposed methods and approaches to the modern international and state requirements;

- consistency of discipline with a mandatory part of the curriculum;

- compliance with the disciplines accepted with the international standards of training, the possibility of student exchange between the foreign universities without loss of quality of the training of these students (academic mobility);

- innovative orientation of the discipline and the opportunities to engage students in innovative activities;

- harmonization of research work with the learning process, which involves the students in research activities;

- the presence of highly qualified teaching staff for the high level discipline training (according to the curriculum);

- the degree of readiness of the students to perceive the training program with the proposed sequence of studying the discipline, and etc.

The proposed criteria for the evaluation of the discipline explicitly are not quantitative, and these indicators need to be translated into a numerical expression for the comparison of educational disciplines on them. This problem can be solved by a group of experts consisting of experienced teachers of university.

After completing such work, a comparison of curriculum can be performed using the analytic hierarchy process $[3,4]$.

\section{Comparison of curriculums using the analytic hierarchy process}

The most important aspect of the quality of the curriculum for a particular university is the development of a coherent vision of the objectives of curriculum and the criteria for their evaluation, shared by all the members of the expert group. This agreement may be executed in a certain hierarchical structure. For this, we must build a hierarchy, starting from the top (objectives - in terms of management), through intermediate levels (characteristics that affect the subsequent levels) to the lowest level, providing a list of alternatives. For this case study, the hierarchical structure is as follows (Fig. 1).

The first level includes the target - "Justification of the selection of the discipline for an optional part of the curriculum", the second level includes the six most important characteristics, and the third level includes the names of disciplines - objects of selection (decision), to be assessed 
on the characteristics of the second level. The proposed list of characteristics evaluate the quality of the following disciplines:

- relevance of the discipline;

- consistency of the discipline with a mandatory part of the curriculum;

- involvement of students in innovation;

- involvement of students in research;

- academic mobility;

- availability of highly qualified faculty personnel for training the discipline (according to the curriculum);

- readiness of students for the perception of the discipline.

In the next phase (after building the hierarchical structure), we construct matrix of pairwise comparisons. The method of pairwise comparisons includes the comparison of the studied factors (alternatives, criteria) with each other. The factors are compared pairwise with respect to their impact ("weight", or "intensity") on their total characterization.

At the same time, for each element adjacent the top level a matrix is built. This element is called dependent (controlled) element regarding the elements of the lower level, since the element of the lower level affects the upper element (though it is subordinate to the target).

In a complete simple hierarchy, any element affects each element adjacent the top level. The elements of each level are compared with each other with respect to their impact on the controlled element. Thus, we get a square matrix of judgments. For each matrix requires judgment $n \cdot(n-1) / 2$ (reciprocals automatically attributed for each pairwise comparison).

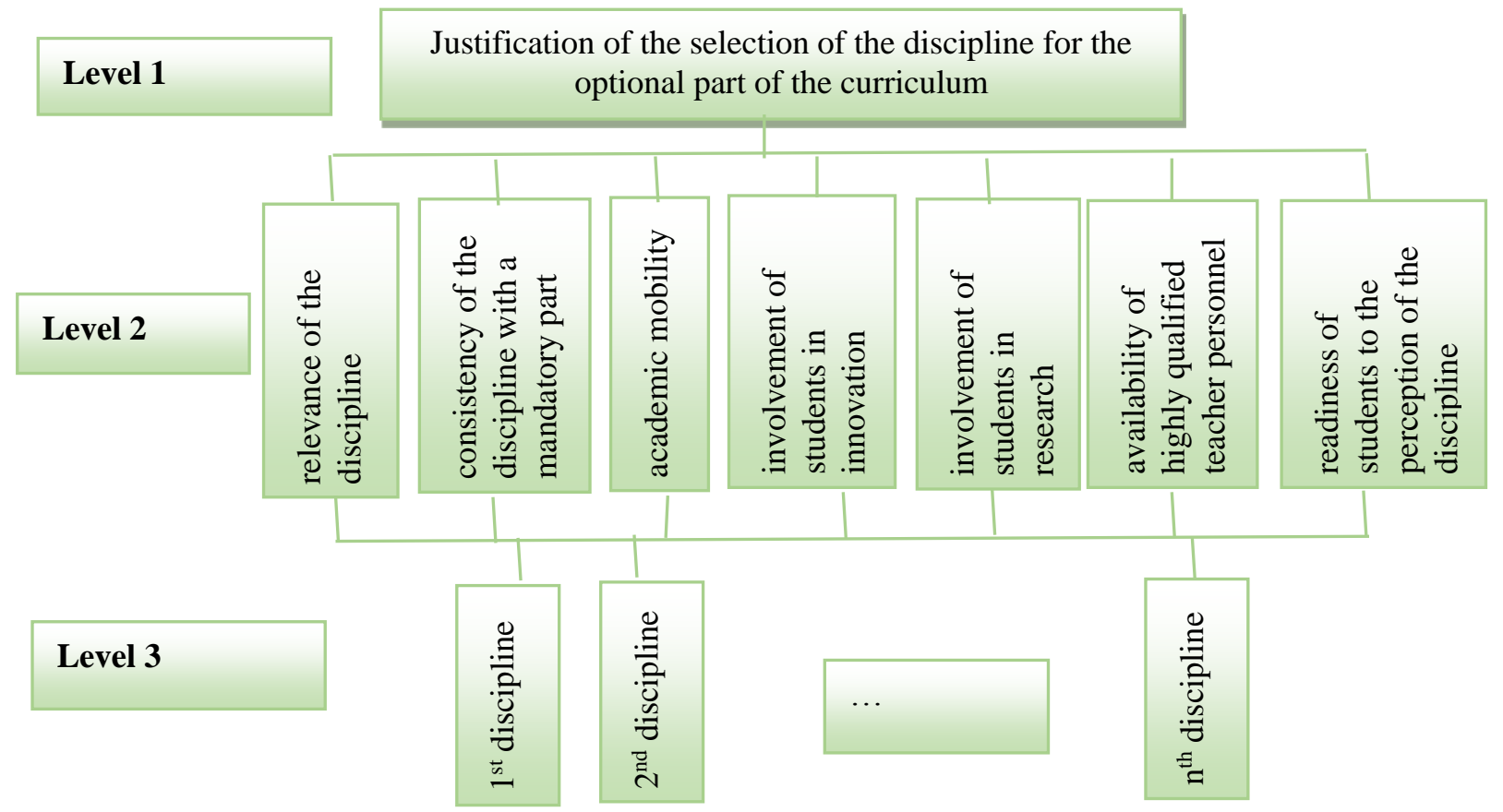

Fig. 1. The hierarchical structure of the discipline selection for the optional part of the curriculum

A1, A2, [...], and An denote the main factors, which are shown in Fig.1. Then, to determine the degree of importance factor, a matrix of pairwise comparisons of the disciplines is completed. A model list of the optional part of the discipline studied at advanced universities of the world, specialized in Computer Science in bachelor degree, is provided below:

- CS1 Introduction to Embedded Systems

- CS2 Distributed Systems

- CS3 Design and development of the user interface 
- CS4 Network Security and Management

- CS5 Information Security Management

- CS6 Operating systems and system programming

- CS7 Software development in emerging platforms

- CS8 Web Engineering

- CS9 Combinatorial and graph-theoretic methods and algorithms

- CS10 Knowledge Discovery and Data Mining

- CS11 Fundamentals of computability and complexity theory

- CS12 Spatio-temporal modeling and simulation

- CS13 Algorithms of Computational Biology

- CS14 Introduction to machine learning

- CS15 Social consequences of computer technology

If we indicate the proportion of impact factor with1 $\mathrm{A}_{\mathrm{i}}$ via $\mathrm{w}_{\mathrm{i}}$ (value, which a decision-maker or an expert puts in accordance with the selected measurement), then, by producing a pairwise comparison of the disciplines, we obtain the matrix elements $a_{i j}=\mathrm{w}_{\mathrm{i}} / \mathrm{w}_{\mathrm{j}}$, which are presented in the Tables 1-7.

The matrices of pairwise comparisons, in this case, are positively defined, back-symmetric, having a rank, which equals to 1 . A feature of back-symmetric matrix of pairwise comparisons is:

1) value, which equals to 1 , must always be on the main diagonal (equal importance, comparable factors, criteria, alternatives), i.e. $a_{i j}=1$ if $\mathrm{i}=\mathrm{j}$;

2) ratio is maintained, which is corresponding to the condition: if the comparison of the $i$-th factor with the $\mathrm{j}$-th factor equals to the value $\mathrm{a}_{\mathrm{ij}}$, then, when comparing the $\mathrm{j}$-th factor with the $\mathrm{i}$-th factor the value $a_{j i}$ will be reverse $a_{i j}$, i.e. $a_{j i}=1 / a_{i j}$.

Tables 1-7 provides an approximate calculation of the values $\mathrm{a}_{\mathrm{ji}}$ when comparing Computer science disciplines by relevant criteria of the Level 2 (Fig.1).

In this case, the rows and columns of the matrix provide the disciplines set out in the initial curriculum. The dimension of the matrix is $15 \times 15$. Filling the matrix of logical relationships is carried out by the columns by the expert teachers, to determine the content of their disciplines. There are 7 (seven) tables of pairwise comparisons - according to the number of criteria (see. Fig.1)

Since the levels of all of the disciplines under the criterion "The relevance of the discipline" are the same, all the coefficients of the first table are aji $=1$, and the weight coefficients are also the same and equal to 0.067 .

Table 1

The matrix of pairwise comparisons of the optional disciplines for Computer science under the criterion of "The relevance of the discipline" of the level 2

\begin{tabular}{|l|c|c|c|c|c|c|c|c|c|c|c|c|c|c|c|c|}
\hline & CS1 & CS2 & CS3 & CS4 & CS5 & CS6 & CS7 & CS8 & CS9 & CS10 & CS11 & CS12 & CS13 & CS14 & CS15 & weight \\
\hline CS1 & 1 & 1 & 1 & 1 & 1 & 1 & 1 & 1 & 1 & 1 & 1 & 1 & 1 & 1 & 1 & 0,067 \\
\hline CS2 & 1 & 1 & 1 & 1 & 1 & 1 & 1 & 1 & 1 & 1 & 1 & 1 & 1 & 1 & 1 & 0,067 \\
\hline CS3 & 1 & 1 & 1 & 1 & 1 & 1 & 1 & 1 & 1 & 1 & 1 & 1 & 1 & 1 & 1 & 0,067 \\
\hline CS4 & 1 & 1 & 1 & 1 & 1 & 1 & 1 & 1 & 1 & 1 & 1 & 1 & 1 & 1 & 1 & 0,067 \\
\hline CS5 & 1 & 1 & 1 & 1 & 1 & 1 & 1 & 1 & 1 & 1 & 1 & 1 & 1 & 1 & 1 & 0,067 \\
\hline CS6 & 1 & 1 & 1 & 1 & 1 & 1 & 1 & 1 & 1 & 1 & 1 & 1 & 1 & 1 & 1 & 0,067 \\
\hline CS7 & 1 & 1 & 1 & 1 & 1 & 1 & 1 & 1 & 1 & 1 & 1 & 1 & 1 & 1 & 1 & 0,067 \\
\hline CS8 & 1 & 1 & 1 & 1 & 1 & 1 & 1 & 1 & 1 & 1 & 1 & 1 & 1 & 1 & 1 & 0,067 \\
\hline CS9 & 1 & 1 & 1 & 1 & 1 & 1 & 1 & 1 & 1 & 1 & 1 & 1 & 1 & 1 & 1 & 0,067 \\
\hline CS10 & 1 & 1 & 1 & 1 & 1 & 1 & 1 & 1 & 1 & 1 & 1 & 1 & 1 & 1 & 1 & 0,067 \\
\hline CS11 & 1 & 1 & 1 & 1 & 1 & 1 & 1 & 1 & 1 & 1 & 1 & 1 & 1 & 1 & 1 & 0,067 \\
\hline CS12 & 1 & 1 & 1 & 1 & 1 & 1 & 1 & 1 & 1 & 1 & 1 & 1 & 1 & 1 & 1 & 0,067 \\
\hline CS13 & 1 & 1 & 1 & 1 & 1 & 1 & 1 & 1 & 1 & 1 & 1 & 1 & 1 & 1 & 1 & 0,067 \\
\hline CS14 & 1 & 1 & 1 & 1 & 1 & 1 & 1 & 1 & 1 & 1 & 1 & 1 & 1 & 1 & 1 & 0,067 \\
\hline CS15 & 1 & 1 & 1 & 1 & 1 & 1 & 1 & 1 & 1 & 1 & 1 & 1 & 1 & 1 & 1 & 0,067 \\
\hline
\end{tabular}


Table 2

The matrix of pairwise comparisons of the optional disciplines for Computer science under the criterion of "Consistency of the discipline with a mandatory part of the curriculum" of the level 2

\begin{tabular}{|l|c|c|c|c|c|c|c|c|c|c|c|c|c|c|c|c|}
\hline & CS1 & CS2 & CS3 & CS4 & CS5 & CS6 & CS7 & CS8 & CS9 & CS10 & CS11 & CS12 & CS13 & CS14 & CS15 & weight \\
\hline CS1 & 1 & $1 / 2$ & $1 / 2$ & $1 / 5$ & $1 / 5$ & 1 & 1 & $1 / 2$ & $1 / 8$ & $1 / 8$ & 1 & 2 & 2 & $1 / 5$ & $1 / 6$ & 0,023 \\
\hline CS2 & 2 & 1 & $1 / 2$ & $1 / 3$ & $1 / 6$ & 2 & 4 & $2 / 5$ & $1 / 5$ & $1 / 5$ & 1 & 3 & 4 & $1 / 2$ & $1 / 5$ & 0,052 \\
\hline CS3 & 2 & 2 & 1 & $1 / 4$ & $1 / 5$ & 1 & 2 & $1 / 3$ & $1 / 5$ & $1 / 5$ & 1 & 3 & 3 & $1 / 2$ & $1 / 5$ & 0,035 \\
\hline CS4 & 5 & 3 & 4 & 1 & $1 / 2$ & 4 & 3 & $1 / 2$ & $1 / 4$ & $1 / 4$ & 3 & 5 & 6 & 2 & $1 / 4$ & 0,075 \\
\hline CS5 & 5 & 6 & 5 & 2 & 1 & 5 & 7 & 1 & 2 & 1 & 4 & 5 & 7 & 2 & $1 / 2$ & 0,134 \\
\hline CS6 & 1 & $1 / 2$ & 1 & $1 / 4$ & $1 / 5$ & 1 & 2 & $1 / 2$ & $1 / 5$ & $1 / 3$ & 2 & 2 & 3 & $1 / 2$ & $1 / 5$ & 0,033 \\
\hline CS7 & 1 & $1 / 4$ & $1 / 2$ & $1 / 3$ & $1 / 7$ & $1 / 2$ & 1 & $1 / 2$ & $1 / 6$ & $1 / 2$ & 1 & 3 & 4 & $1 / 2$ & $1 / 4$ & 0,028 \\
\hline CS8 & 2 & $5 / 2$ & 3 & 2 & 1 & 2 & 2 & 1 & $1 / 4$ & $1 / 2$ & 2 & 4 & 6 & 2 & $1 / 4$ & 0,074 \\
\hline CS9 & 8 & 5 & 5 & 4 & $1 / 2$ & 5 & 6 & 4 & 1 & $1 / 2$ & 5 & 7 & 7 & 3 & $1 / 2$ & 0,144 \\
\hline CS10 & 8 & 5 & 5 & 4 & 1 & 3 & 2 & 2 & 2 & 1 & 3 & 3 & 5 & 3 & 1 & 0,133 \\
\hline CS11 & 1 & 1 & 1 & $1 / 3$ & $1 / 4$ & $1 / 2$ & 1 & $1 / 2$ & $1 / 5$ & $1 / 3$ & 1 & 1 & 2 & $1 / 2$ & $1 / 4$ & 0,029 \\
\hline CS12 & $1 / 2$ & $1 / 3$ & $1 / 3$ & $1 / 5$ & $1 / 5$ & $1 / 2$ & $1 / 3$ & $1 / 4$ & $1 / 7$ & $1 / 3$ & 1 & 1 & $1 / 3$ & $1 / 3$ & $1 / 5$ & 0,017 \\
\hline CS13 & $1 / 2$ & $1 / 4$ & $1 / 3$ & $1 / 6$ & $1 / 7$ & $1 / 3$ & $1 / 4$ & $1 / 6$ & $1 / 7$ & $1 / 5$ & $1 / 2$ & 3 & 1 & $1 / 4$ & $1 / 7$ & 0,015 \\
\hline CS14 & 5 & 2 & 2 & $1 / 2$ & $1 / 2$ & 2 & 2 & $1 / 2$ & $1 / 3$ & $1 / 3$ & 2 & 3 & 4 & 1 & $1 / 2$ & 0,059 \\
\hline CS15 & 6 & 5 & 5 & 4 & 2 & 5 & 4 & 4 & 3 & 1 & 4 & 5 & 7 & 2 & 1 & 0,163 \\
\hline
\end{tabular}

Table 3

The matrix of pairwise comparisons of the optional disciplines for Computer science under the criterion of "Academic mobility" of the level 2

\begin{tabular}{|l|c|c|c|c|c|c|c|c|c|c|c|c|c|c|c|c|}
\hline & CS1 & CS2 & CS3 & CS4 & CS5 & CS6 & CS7 & CS8 & CS9 & CS10 & CS11 & CS12 & CS13 & CS14 & CS15 & weight \\
\hline CS1 & 1 & 1 & 1 & $1 / 4$ & $1 / 5$ & $1 / 2$ & $1 / 2$ & $1 / 6$ & 1 & $1 / 6$ & 1 & 1 & 2 & $1 / 5$ & $1 / 6$ & 0,026 \\
\hline CS2 & 1 & 1 & 1 & $1 / 2$ & $1 / 2$ & 3 & $1 / 2$ & 1 & 3 & 1 & 5 & 3 & 4 & 2 & $1 / 2$ & 0,07 \\
\hline CS3 & 1 & 1 & 1 & $1 / 2$ & $2 / 5$ & $1 / 4$ & 2 & $1 / 2$ & 2 & $1 / 4$ & $1 / 2$ & 4 & 4 & $1 / 2$ & $1 / 6$ & 0,04 \\
\hline CS4 & 4 & 2 & 2 & 1 & 1 & 4 & 2 & $2 / 5$ & 2 & $1 / 2$ & 4 & 4 & 4 & 2 & $1 / 2$ & 0,09 \\
\hline CS5 & 5 & 2 & $5 / 2$ & 1 & 1 & 4 & 2 & 2 & 5 & 1 & 4 & 5 & 8 & 3 & 1 & 0,132 \\
\hline CS6 & 2 & $1 / 3$ & 4 & $1 / 4$ & $1 / 4$ & 1 & 1 & $1 / 2$ & $1 / 2$ & $1 / 6$ & 2 & 2 & 4 & 2 & $1 / 5$ & 0,043 \\
\hline CS7 & 2 & 2 & $1 / 2$ & $1 / 2$ & $1 / 2$ & 1 & 1 & $1 / 2$ & 2 & $1 / 4$ & 2 & 2 & 4 & 1 & $1 / 4$ & 0,05 \\
\hline CS8 & 6 & 1 & 2 & $5 / 2$ & $1 / 2$ & 2 & 2 & 1 & 4 & 1 & 3 & 5 & 6 & 2 & 1 & 0,108 \\
\hline CS9 & 1 & $1 / 3$ & $1 / 2$ & $1 / 2$ & $1 / 5$ & 2 & $1 / 2$ & $1 / 4$ & 1 & $1 / 4$ & 2 & $1 / 2$ & $1 / 2$ & $1 / 4$ & $1 / 6$ & 0,026 \\
\hline CS10 & 6 & 1 & 4 & 2 & 1 & 6 & 4 & 1 & 4 & 1 & 4 & 6 & 4 & 4 & 1 & 0,137 \\
\hline CS11 & 1 & $1 / 5$ & 2 & $1 / 4$ & $1 / 4$ & $1 / 2$ & $1 / 2$ & $1 / 3$ & $1 / 2$ & $1 / 4$ & 1 & 1 & 1 & $1 / 4$ & $1 / 6$ & 0,025 \\
\hline CS12 & 1 & $1 / 3$ & $1 / 4$ & $1 / 4$ & $1 / 5$ & $1 / 2$ & $1 / 2$ & $1 / 5$ & $1 / 2$ & $1 / 6$ & 1 & 1 & 1 & $1 / 2$ & $1 / 8$ & 0,021 \\
\hline CS13 & $1 / 2$ & $1 / 4$ & $1 / 5$ & $1 / 4$ & $1 / 8$ & $1 / 4$ & $1 / 4$ & $1 / 6$ & $1 / 4$ & $1 / 8$ & $1 / 2$ & 1 & 1 & $1 / 4$ & $1 / 8$ & 0,014 \\
\hline CS14 & 5 & $1 / 2$ & 2 & $1 / 2$ & $1 / 3$ & $1 / 2$ & 1 & $1 / 2$ & 4 & $1 / 4$ & 4 & 2 & 4 & 1 & $1 / 2$ & 0,057 \\
\hline CS15 & 6 & 2 & 6 & 2 & 1 & 5 & 4 & 1 & 6 & 1 & 6 & 8 & 8 & 2 & 1 & 0,158 \\
\hline
\end{tabular}

Table 4

The matrix of pairwise comparisons of the optional disciplines for Computer science under the criterion of "Involvement of students in innovation" of the level 2

\begin{tabular}{|l|c|c|c|c|c|c|c|c|c|c|c|c|c|c|c|c|}
\hline & CS1 & CS2 & CS3 & CS4 & CS5 & CS6 & CS7 & CS8 & CS9 & CS10 & CS11 & CS12 & CS13 & CS14 & CS15 & weight \\
\hline CS1 & 1 & $1 / 3$ & $1 / 3$ & $1 / 2$ & $1 / 2$ & 1 & $1 / 2$ & $1 / 4$ & 4 & $1 / 4$ & 1 & 1 & $1 / 2$ & $1 / 2$ & $1 / 2$ & 0,034 \\
\hline CS2 & 3 & 1 & 1 & $1 / 2$ & $1 / 2$ & 2 & $1 / 2$ & $1 / 5$ & 2 & $1 / 5$ & 2 & 2 & 1 & 1 & $1 / 2$ & 0,05 \\
\hline CS3 & 3 & 1 & 1 & $1 / 2$ & $2 / 5$ & 3 & 1 & $2 / 5$ & 5 & $1 / 2$ & 3 & 1 & $1 / 2$ & 1 & 3 & 0,066 \\
\hline CS4 & 2 & 2 & 2 & 1 & 1 & 2 & 1 & $1 / 1$ & 4 & 1 & 4 & 1 & 1 & 2 & $1 / 2$ & 0,079 \\
\hline CS5 & 2 & 2 & $5 / 2$ & 1 & 1 & 4 & 2 & 1 & 4 & 1 & 4 & 2 & 2 & 2 & 1 & 0,106 \\
\hline
\end{tabular}


Problems of information technology, 2016, №1, 47-54

\begin{tabular}{|l|c|c|c|c|c|c|c|c|c|c|c|c|c|c|c|c|}
\hline CS6 & 1 & $1 / 2$ & $1 / 3$ & $1 / 2$ & $1 / 4$ & 1 & $1 / 3$ & $1 / 4$ & 2 & $1 / 6$ & 1 & $1 / 2$ & $1 / 3$ & $1 / 3$ & $1 / 5$ & 0,026 \\
\hline CS7 & 2 & 2 & 1 & 1 & $1 / 2$ & 3 & 1 & $1 / 2$ & 3 & $1 / 4$ & 2 & 2 & 1 & $1 / 2$ & $1 / 2$ & 0,06 \\
\hline CS8 & 4 & 5 & $5 / 2$ & 2 & 1 & 4 & 2 & 1 & 4 & 1 & 3 & 3 & 2 & 2 & 1 & 0,125 \\
\hline CS9 & $1 / 4$ & $1 / 2$ & $1 / 5$ & $1 / 4$ & $1 / 4$ & $1 / 2$ & $1 / 3$ & $1 / 4$ & 1 & $1 / 5$ & 1 & $1 / 2$ & $1 / 2$ & $1 / 4$ & $1 / 4$ & 0,02 \\
\hline CS10 & 4 & 5 & 2 & 1 & 1 & 6 & 4 & 1 & 5 & 1 & 4 & 3 & 3 & 2 & 2 & 0,141 \\
\hline CS11 & 1 & $1 / 2$ & $1 / 3$ & $1 / 4$ & $1 / 4$ & 1 & $1 / 2$ & $1 / 3$ & 1 & $1 / 4$ & 1 & $1 / 2$ & $1 / 2$ & $1 / 2$ & $1 / 3$ & 0,028 \\
\hline CS12 & 1 & $1 / 2$ & 1 & 1 & $1 / 2$ & 2 & $1 / 2$ & $1 / 3$ & 2 & $1 / 3$ & 2 & 1 & 1 & $1 / 2$ & $1 / 2$ & 0,045 \\
\hline CS13 & 2 & 1 & 2 & 1 & $1 / 2$ & 3 & 1 & $1 / 2$ & 2 & $1 / 3$ & 2 & 1 & 1 & 1 & 1 & 0,063 \\
\hline CS14 & 2 & 1 & 1 & $1 / 2$ & $1 / 2$ & 3 & 2 & $1 / 2$ & 4 & $1 / 2$ & 2 & 2 & 1 & 1 & $1 / 4$ & 0,062 \\
\hline CS15 & 2 & 2 & $1 / 3$ & 2 & 1 & 5 & 2 & 1 & 4 & $1 / 2$ & 3 & 2 & 1 & 4 & 1 & 0,093 \\
\hline
\end{tabular}

$\lambda_{\max }=15,94 ; \mathrm{HS}=0,067$

Table 5

The matrix of pairwise comparisons of the optional disciplines for Computer science under the criterion of "Involvement of students in research" of the level 2

\begin{tabular}{|l|c|c|c|c|c|c|c|c|c|c|c|c|c|c|c|c|}
\hline & CS1 & CS2 & CS3 & CS4 & CS5 & CS6 & CS7 & CS8 & CS9 & CS10 & CS11 & CS12 & CS13 & CS14 & CS15 & weight \\
\hline CS1 & 1 & $1 / 2$ & $1 / 4$ & $1 / 4$ & $1 / 4$ & 1 & 1 & $1 / 3$ & 2 & $1 / 6$ & 1 & 1 & $1 / 4$ & $1 / 4$ & $1 / 3$ & 0,027 \\
\hline CS2 & 2 & 1 & $1 / 2$ & $1 / 2$ & 3 & 4 & 3 & $1 / 2$ & 5 & $1 / 3$ & 2 & $1 / 2$ & $1 / 2$ & 1 & $1 / 2$ & 0,061 \\
\hline CS3 & 4 & 2 & 1 & $1 / 3$ & $1 / 3$ & 1 & 1 & $1 / 4$ & 2 & $1 / 4$ & $1 / 3$ & $1 / 3$ & $1 / 4$ & $1 / 4$ & $1 / 2$ & 0,033 \\
\hline CS4 & 4 & 2 & 3 & 1 & 1 & 4 & 2 & 1 & 4 & 1 & 2 & 2 & 1 & 2 & 1 & 0,1 \\
\hline CS5 & 4 & $1 / 3$ & 3 & 1 & 1 & 4 & 4 & 1 & 4 & 1 & 2 & 2 & 1 & 1 & 1 & 0,089 \\
\hline CS6 & 1 & $1 / 4$ & 1 & $1 / 4$ & $1 / 4$ & 1 & 1 & $1 / 4$ & 2 & $1 / 4$ & $1 / 2$ & $1 / 4$ & $1 / 4$ & $1 / 4$ & $1 / 2$ & 0,025 \\
\hline CS7 & 1 & $1 / 3$ & 1 & $1 / 2$ & $1 / 4$ & 1 & 1 & $1 / 4$ & 2 & $1 / 4$ & 1 & 1 & $1 / 2$ & $1 / 2$ & $1 / 2$ & 0,034 \\
\hline CS8 & 3 & 2 & 4 & 1 & 1 & 4 & 4 & 1 & 4 & 1 & 4 & 4 & 1 & 2 & 1 & 0,115 \\
\hline CS9 & $1 / 2$ & $1 / 5$ & $1 / 2$ & $1 / 4$ & $1 / 4$ & $1 / 2$ & $1 / 2$ & $1 / 4$ & 1 & $1 / 4$ & $1 / 3$ & $1 / 4$ & $1 / 4$ & $1 / 4$ & $1 / 6$ & 0,018 \\
\hline CS10 & 6 & 3 & 4 & 1 & 1 & 4 & 4 & 1 & 4 & 1 & 6 & 3 & 1 & 1 & 1 & 0,119 \\
\hline CS11 & 1 & 2 & 3 & $1 / 2$ & $1 / 2$ & 2 & 1 & $1 / 4$ & 3 & $1 / 6$ & 1 & $1 / 2$ & $1 / 4$ & $1 / 4$ & $1 / 3$ & 0,039 \\
\hline CS12 & 1 & 2 & 3 & $1 / 2$ & $1 / 2$ & 4 & 1 & $1 / 4$ & 4 & $1 / 3$ & 2 & 1 & $1 / 4$ & $1 / 2$ & $1 / 3$ & 0,05 \\
\hline CS13 & 4 & 2 & 4 & 1 & 1 & 4 & 2 & 1 & 4 & 1 & 4 & 4 & 1 & 1 & 1 & 0,106 \\
\hline CS14 & 4 & 1 & 4 & $1 / 2$ & 1 & 4 & 2 & $1 / 2$ & 4 & 1 & 4 & 2 & 1 & 1 & 3 & 0,095 \\
\hline CS15 & 3 & 2 & 2 & 1 & 1 & 2 & 2 & 1 & 6 & 1 & 3 & 3 & 1 & $1 / 3$ & 1 & 0,088 \\
\hline
\end{tabular}

Table 6

The matrix of pairwise comparisons of the optional disciplines for Computer science under the criterion of "Availability of highly qualified teacher personnel" of the level 2

\begin{tabular}{|l|c|c|c|c|c|c|c|c|c|c|c|c|c|c|c|c|}
\hline & CS1 & CS2 & CS3 & CS4 & CS5 & CS6 & CS7 & CS8 & CS9 & CS10 & CS11 & CS12 & CS13 & CS14 & CS15 & weight \\
\hline CS1 & 1 & 1 & $1 / 2$ & $1 / 4$ & $1 / 6$ & 1 & 1 & $1 / 4$ & $1 / 6$ & $1 / 4$ & 1 & 2 & 2 & $1 / 2$ & $1 / 6$ & 0,027 \\
\hline CS2 & 1 & 1 & 1 & $1 / 4$ & $1 / 6$ & 2 & 2 & $1 / 2$ & $1 / 4$ & $1 / 4$ & 1 & 2 & 4 & 1 & $1 / 4$ & 0,051 \\
\hline CS3 & 2 & 1 & 1 & $1 / 4$ & $1 / 6$ & 1 & 1 & $1 / 4$ & $1 / 4$ & $1 / 4$ & 1 & 1 & 1 & $1 / 4$ & $1 / 4$ & 0,027 \\
\hline CS4 & 4 & 4 & 4 & 1 & $1 / 2$ & 4 & 4 & 1 & $1 / 2$ & 1 & 2 & 4 & 4 & 4 & 1 & 0,1 \\
\hline CS5 & 6 & 6 & 6 & 2 & 1 & 4 & 4 & 1 & $1 / 2$ & 1 & 4 & 4 & 4 & 2 & 1 & 0,119 \\
\hline CS6 & 1 & $1 / 2$ & 1 & $1 / 4$ & $1 / 4$ & 1 & $1 / 2$ & $1 / 6$ & $1 / 6$ & $1 / 6$ & 1 & 2 & 1 & $1 / 2$ & $1 / 6$ & 0,024 \\
\hline CS7 & 1 & $1 / 2$ & 1 & $1 / 4$ & $1 / 4$ & 2 & 1 & $1 / 4$ & $1 / 8$ & $1 / 6$ & 1 & 1 & 1 & $1 / 2$ & $1 / 4$ & 0,026 \\
\hline CS8 & 4 & 2 & 4 & 1 & 1 & 6 & 4 & 1 & $1 / 2$ & $1 / 2$ & 4 & 4 & 4 & 2 & $1 / 2$ & 0,094 \\
\hline CS9 & 6 & 4 & 4 & 2 & 2 & 6 & 8 & 2 & 1 & 2 & 6 & 8 & 6 & 4 & 1 & 0,169 \\
\hline CS10 & 4 & 4 & 4 & 1 & 1 & 6 & 6 & 2 & $1 / 2$ & 1 & 4 & 5 & 5 & 4 & 1 & 0,125 \\
\hline CS11 & 1 & 1 & 1 & $1 / 2$ & $1 / 4$ & 1 & 1 & $1 / 4$ & $1 / 6$ & $1 / 4$ & 1 & 1 & 1 & $1 / 2$ & $1 / 6$ & 0,027 \\
\hline CS12 & $1 / 2$ & $1 / 2$ & 1 & $1 / 4$ & $1 / 4$ & $1 / 2$ & 1 & $1 / 4$ & $1 / 8$ & $1 / 5$ & 1 & 1 & 1 & $1 / 2$ & $1 / 6$ & 0,022 \\
\hline CS13 & $1 / 2$ & $1 / 4$ & 1 & $1 / 4$ & $1 / 4$ & 1 & 1 & $1 / 4$ & $1 / 6$ & $1 / 5$ & 1 & 1 & 1 & $1 / 4$ & $1 / 6$ & 0,021 \\
\hline CS14 & 2 & 1 & 4 & $1 / 4$ & $1 / 2$ & 2 & 2 & $1 / 2$ & $1 / 4$ & $1 / 4$ & 2 & 2 & 4 & 1 & $1 / 2$ & 0,051 \\
\hline CS15 & 6 & 4 & 4 & 1 & 1 & 6 & 4 & 2 & 1 & 1 & 6 & 6 & 6 & 2 & 1 & 0,131 \\
\hline
\end{tabular}

$\lambda_{\max }=15,59 ; \mathrm{HS}=0,042$ 
Table 7

The matrix of pairwise comparisons of the optional disciplines for Computer science under the criterion of "Readiness of students for the perception of the discipline" of the level 2

\begin{tabular}{|l|c|c|c|c|c|c|c|c|c|c|c|c|c|c|c|c|}
\hline & CS1 & CS2 & CS3 & CS4 & CS5 & CS6 & CS7 & CS8 & CS9 & CS10 & CS11 & CS12 & CS13 & CS14 & CS15 & weight \\
\hline CS1 & 1 & 1 & $1 / 2$ & $1 / 4$ & $1 / 6$ & 1 & 1 & $1 / 4$ & $1 / 6$ & $1 / 6$ & 1 & 1 & 1 & $1 / 2$ & $1 / 6$ & 0,024 \\
\hline CS2 & 1 & 1 & 2 & $1 / 4$ & $1 / 4$ & 2 & 2 & $2 / 5$ & $1 / 4$ & $1 / 2$ & 2 & 4 & 2 & 2 & $1 / 4$ & 0,05 \\
\hline CS3 & 2 & $1 / 2$ & 1 & $1 / 6$ & $1 / 8$ & 1 & 1 & $1 / 4$ & $1 / 6$ & $1 / 5$ & 1 & 1 & 1 & $1 / 2$ & $1 / 5$ & 0,023 \\
\hline CS4 & 4 & 4 & 5 & 1 & $1 / 2$ & 4 & 5 & 1 & $1 / 2$ & $1 / 2$ & 4 & 4 & 6 & 2 & $1 / 2$ & 0,095 \\
\hline CS5 & 6 & 4 & 8 & 2 & 1 & 6 & 6 & 1 & 1 & 1 & 5 & 5 & 6 & 2 & 1 & 0,137 \\
\hline CS6 & 1 & $1 / 2$ & 1 & $1 / 4$ & $1 / 6$ & 1 & 1 & $1 / 4$ & $1 / 6$ & $1 / 5$ & 1 & 1 & 1 & 1 & $1 / 5$ & 0,025 \\
\hline CS7 & 1 & $1 / 2$ & 1 & $1 / 5$ & $1 / 6$ & 1 & 1 & $1 / 5$ & $1 / 6$ & $1 / 5$ & 1 & 1 & 1 & $1 / 2$ & $1 / 5$ & 0,023 \\
\hline CS8 & 4 & $5 / 2$ & 4 & 1 & 1 & 4 & 5 & 1 & 1 & 1 & 4 & 4 & 4 & 2 & $1 / 4$ & 0,098 \\
\hline CS9 & 6 & 4 & 6 & 2 & 1 & 6 & 6 & 1 & 1 & 2 & 5 & 5 & 6 & 2 & $1 / 2$ & 0,135 \\
\hline CS10 & 6 & 2 & 5 & 2 & 1 & 5 & 5 & 1 & $1 / 2$ & 1 & 4 & 5 & 4 & 2 & $1 / 4$ & 0,103 \\
\hline CS11 & 1 & $1 / 2$ & 1 & $1 / 4$ & $1 / 5$ & 1 & 1 & $1 / 4$ & $1 / 5$ & $1 / 4$ & 1 & 1 & 2 & $1 / 2$ & $1 / 5$ & 0,026 \\
\hline CS12 & 1 & $1 / 4$ & 1 & 1 & $1 / 4$ & $1 / 5$ & 1 & 1 & $1 / 4$ & $1 / 5$ & $1 / 5$ & 1 & 1 & 2 & $1 / 5$ & 0,024 \\
\hline CS13 & 1 & $1 / 2$ & 1 & $1 / 6$ & $1 / 6$ & 1 & 1 & $1 / 4$ & $1 / 6$ & $1 / 4$ & $1 / 2$ & $1 / 2$ & 1 & $1 / 2$ & $1 / 6$ & 0,021 \\
\hline CS14 & 2 & $1 / 2$ & 2 & $1 / 2$ & $1 / 2$ & 1 & 2 & $1 / 2$ & $1 / 2$ & $1 / 2$ & 2 & 2 & 2 & 1 & $1 / 5$ & 0,051 \\
\hline CS15 & 6 & 4 & 5 & 2 & 1 & 5 & 5 & 4 & 2 & 4 & 5 & 5 & 6 & 5 & 1 & 0,174 \\
\hline
\end{tabular}

Based on the values of the vector components of the generalized priority, the option (discipline) is selected, which has a maximum value (weight) in the found vector.

Obviously, two questions araise:

1) how to determine the importance of the impact of each factor on the case study?;

2) how to determine the reliability of the result?

To answer these questions, the calculations are made by the definition of the relative priority of the factors and values of consistency of the evaluations that the expert puts.

The reliability of obtained results provides consistency of the evaluations. At the same time, the following statement includes: positive back-symmetric matrix is consistent and only if the order of the matrix and its largest eigenvalue match up, i.e., $\lambda_{\max }=n$.

Eigenvector of the matrix of paired comparisons, called a vector of priorities, provides the arrangement of the priorities, and its eigenvalues define the measure of the judgments consistency. The method of finding the vector $w$ is based on one of the judgments of linear algebra [5] - required vector is an eigenvector of pairwise comparisons corresponding to the maximum eigenvalue $\left(\lambda_{\max }\right)$. In this case, $\lambda_{\max }$ is found, and then, the vector equation $\mathrm{A}^{*} \mathrm{w}=\lambda_{\max }{ }^{*} \mathrm{w}$ is solved.

After implementing all pairwise comparisons and obtaining the data on eigenvalue and eigenvector, the consistency can be defined. To do this, we calculate the value called the index of consistency by the following formula, by using the deviation of the maximum eigenvalue from the dimension of the matrix:

$H S=\frac{\lambda_{\max }-n}{n-1}$, where $\mathrm{n}-$ denotes the dimension of the matrix (Tables 2-7 show the indices of consistency, they do not exceed the value 0.10 ).

We note that a number, not exceeding $10 \%$, is acceptable for the overall coherence (OC). Otherwise, the appropriate matrix must be re-evaluated.

Based on pairwise comparisons, we set the list of disciplines - the weights that are above the average $(>0.05)$ in all seven tables:

- CS2 Distributed Systems

- CS4 Network Security and Management

- CS5 Information Security Management 
- CS8 Web Engineering

- CS9 Combinatorial and graph-theoretic methods and algorithms

- CS10 Knowledge Discovery and Data Mining

- CS14 Introduction to machine learning

- CS15 Social consequences of computer technology

The list of disciplines (an optional part of the curriculum) is recommended for the study of Computer Science for the bachelor's degree at the universities of Azerbaijan.

\section{Conclusion}

The analysis of the selection of optimal curriculum has shown that taking into account the nature and correct comparison and evaluation of the educational disciplines requires a method for the mathematical processing of the qualitative criteria. The most appropriate method, in this case, is the analytic hierarchy process.

Thus, such constructed hierarchy, on the one hand, is a tool for comparing various curriculums in the field of IT, and on the other hand, it may be subject to discussion of the objectives and criteria of the IT education for all interested parties.

To optimize the curriculum, based on the values of the vector components of the generalized priority, we select the option with the maximum value. Thus, the analysis using the analytic hierarchy process shows the possibility of objectification of the option and decision-making in such a complex and conceptual problem as the optimization of the curriculum.

\section{References}

1. Baranova N.A. "Designing the content of uninterrupted education using the expert system". Monograph, Izhevsk, 2008. $126 \mathrm{p}$.

2. Standards and Guidelines for Quality Assurance in the European Higher Education Area, Report of European Association for Quality Assurance in Higher Education, 2005. www.enqa.eu/files/BergenReport210205.pdf

3. Saaty T. Decision-maikng. Analytic hierarchy process. M.: Radio and Communications, 1993, $278 \mathrm{p}$.

4. Saaty T.L., Keris K.P. Analytic planning. System organisation. Trans. from English. ed. Ushakova I.A., M.: Radio and Communications, 1991, 244 p.

5. Umnov A.E. Analytic geometry and linear algebra, Moscow, 2011, 543 p. 